\title{
Hydraulic Dynamic Calculation and Simulation
}

\author{
Costanza Aricò (D)
}

Citation: Aricò, C. Hydraulic Dynamic Calculation and Simulation. Water 2021, 13, 1234. https:// doi.org/10.3390/w13091234

Received: 9 April 2021

Accepted: 25 April 2021

Published: 29 April 2021

Publisher's Note: MDPI stays neutral with regard to jurisdictional claims in published maps and institutional affiliations.

Copyright: (C) 2021 by the author. Licensee MDPI, Basel, Switzerland. This article is an open access article distributed under the terms and conditions of the Creative Commons Attribution (CC BY) license (https:// creativecommons.org/licenses/by/ $4.0 /)$.
Department of Engineering, University of Palermo, 90133 Palermo, Italy; costanza.arico@unipa.it

Hydraulic dynamics is emerging as a basic concept with applications and important implications for many industrial, civil, and environmental engineering problems. " $\mathrm{Hy}$ draulic Dynamic Calculation and Simulation" can be regarded as an important tool for the analysis and prediction of many physical processes and their related problems, as well as for decision-making with regard to mitigative measures.

In the present Special Issue, we have collected 20 research papers [1-20] which are grouped in two main general categories, i.e., civil/environmental applications [1-10] and industrial applications [11-20]. Most of the presented studies regarding industrial applications propose innovative aspects with implications for many environmental processes, while most of the civil/environmental studies present aspects strongly interconnected with "industrial" issues.

For the first main category, regarding civil and environmental applications, we received numerical and experimental contributions related to:

- The investigation of the interaction between fluid and structures (e.g., real cases of dam-break flows or hydraulic jumps over specific macro-roughness) and research on fluid-driven fractures; and

- Studies on fluids and transport problems in lakes, estuaries, and coastal zones and studies on the density of jet currents of pollutant emissions.

In the second main category, regarding industrial applications, we received numerical and experimental contributions related to:

- The investigation of renewable energy recovery systems or energy-saving strategies (e.g., the optimization and improvement studies of PAT, crossflow, and river turbines, as well as piped or capsule hydraulic transportation); and

- Studies on the fundamental structural aspects of hydraulic machineries (e.g., the application of vortex pumps in sewer systems, and the hydraulic design of submersible pumps).

The authors of [1] investigated the hydraulic characteristics of the dam discharge flow. They employed Reynolds-averaged Navier-Stokes equations with the RNG k- $\varepsilon$ eddy viscosity model for turbulence closure, as well as the volume of fluid method. An urban flood experiment and the field measurement records were used to validate the model's accuracy. The dam satisfied the safety requirements under different water levels, but attention should be paid to the dam foundation, especially around the incident points of the discharge flow.

The aim of paper [2] was to investigate the ability of some numerical 2D and 3D models to simulate flood waves in the presence of an isolated building or an array of buildings in an inundated area. Firstly, a 2D finite-volume method numerical model was proposed to solve 2D shallow-water equations (2D-SWEs) on structured mesh. Secondly, a 3D commercial CFD software package was selected, which contained a Flow-3D model with two turbulent models: Reynolds-averaged Navier-Stokes (RANs) with a renormalized group (RNG), and large-eddy simulation (LES). According to the numerical results regarding the impact force on an obstruction due to dam-break flow, the 3D solution was much better than the 2D one.

In the work presented in [3], the importance of a non-hydrostatic hydrodynamic approach was highlighted, since in some free-surface flow processes (e.g., lakes, estu- 
aries, reservoirs, and coastal zones), vertical velocities are relevant. A nonhydrostatic hydrodynamic approach is not free of numerical diffusion. The combined use of highresolution schemes in coupled nonhydrostatic hydrodynamic and solute transport models is a promising alternative to minimize these numerical issues.

When the ratio of vertical to horizontal motion scales is not small, a non-hydrostatic approximation is needed to accurately simulate 3D free-surface flows in large aquatic ecosystems. The authors in [4] proposed a novel free-surface boundary condition based on a fictional sublayer at the free surface (FSFS). They applied the FSFS approach using finite difference numerical discretization with a fractional step framework and compared the classic boundary condition approach (CBA) and the FSFS approach using two numerical experiments.

The authors of [5] presented a novel 3D coupled computational fluid dynamics (CFD)discrete element method (DEM) model by coupling two software programs, OpenFOAM and PFC3D, to solve problems related to fluid-particle interaction systems. Two classic geo-mechanics problems (where analytical solutions are available) were first presented to validate the coupled CFD-DEM. Then, the solver was applied to investigate the flow characteristics of a particle heap subjected to the effects of water inside a pipe under different conditions. The results showed that particle size and pipe inclination angle can significantly affect the particle flow morphology, total kinetic energy, and erosion rate for mono-sized particles, whereas polydisperse particles had a slight effect. The proposed numerical approach could provide valuable references for solving soil erosion or bridge scour problems.

In paper [6], numerical simulations were conducted in order to understand the role of wave-current interactions in wave deformation. The wave-current interaction mechanisms, wave reflection and energy loss due to currents, effect of incident conditions on wave-current interactions, advection-diffusion characteristics of saltwater, and the effect of density currents on wave-current interactions were discussed. In addition, the effect of saltwater-freshwater density on wave-current interactions was investigated under a hypopycnal flow field via numerical model testing. These results improve our understanding of hydrodynamic phenomena in estuaries in which saltwater-freshwater and wave-current pairs coexist.

The authors of paper [7] investigated the realistic simulation of ocean scenes, improving a smoothed particle hydrodynamics (SPH) model. The solver combines nonlinear constant density constraints and a divergence-free velocity field constraint. The proposed model presents high solution efficiency, large time steps, and strong numerical stability. The authors introduced a unified boundary handling model to simulate coupling scenes. The model samples the boundary geometry as particles by means of single-layer nonuniform sampling. The contribution of the boundary particles is taken into account when the physical quantities of fluid particles are computed. The unified model handles complex geometries, providing a theoretical reference for reliable maritime scenarios.

Paper [8] investigated the water inrush caused by hydrosplitting, an important disaster in the engineering of underground tunnels. The propagation of fluid-driven fractures based on an improved discrete element fluid-solid coupling method was modeled. The authors first investigated the interactions between hydrosplitting fractures (HFs) and preexisting weak planes (WPs) with different angles, assuming water pressure in the initial fracture. Then, the authors analyzed the influence of the in situ stress ratio and the property of WPs, and the corresponding critical pressure values of different interactions were calculated. Lastly, the maximum principal stress and maximum shear stress variation inside the pieces were simulated.

A numerical study on the interaction between vertical turbulent jets and the pycnocline was carried out in [9] to study the problems of jet emissions through large eddy simulation (LES). A trigonometric function disturbance (TFD) method was proposed to ensure the velocity distribution of the jet in the horizontal plane yield to Gaussian profile. Numerical simulations were performed in the range of $1.11<\operatorname{Frp}<4.77$, corresponding to 
$1393<$ Rep < 5979, with the Froude number (Frp) and the Reynolds number (Rep) defined at the entrance of pycnocline. Coherent structure and internal waves were observed at the pycnocline during the process of jets impinging. After the impingement, destratification effects could be also observed. A fitting formula of the radial momentum flux dissipation rate, used to describe the decay of energy of the impinging jets, was obtained by means of dimensionless analysis.

The objective of paper [10] was to numerically investigate the effects of triangular macro-roughness on the characteristics of submerged jump, e.g., the longitudinal profile of streamlines, flow patterns in the cavity region, horizontal velocity profiles, streamwise velocity distribution, thickness of the inner layer, bed shear stress coefficient, turbulent kinetic energy (TKE), and energy loss. Different macroroughness arrangements and various inlet Froude numbers $(1.7<\mathrm{Fr}<9.3)$ were considered. Experimental data from the literature were considered to verify the accuracy and reliability of the numerical results.

The use of a flow discharge-measuring device in irrigated areas was investigated in [11]. This is the key to developing water-saving irrigation techniques. A new plate measuring device of flow discharge for a U-channel was designed. The hydraulic characteristics of the flow discharge measurement process using this device were simulated and experimentally verified, adopting a renormalization group $k-\varepsilon$ turbulence model based on Flow-3D software. According to the results, the flow characteristics were consistent with the experimental results.

Paper [12] studied the use of pumps working as turbines (PATs) to improve the energy efficiency of water networks. Analyses of water systems using simulation software and/or optimization algorithms require accurate characteristic curves (head and efficiency) of the machines. The knowledge of the best efficiency point (BEP) as a turbine is one of the major limitations in the use of PATs. New equations to estimate the BEP of the PAT were presented and also used to predict the characteristic curves, comparing the results with the rest of the published methods. The new proposal increased the accuracy of the error ellipse using an experimental database of 181 different PATs.

The pressure reduction valves (PRVs) in pressurized water distribution systems increased water usage efficiency, while at the same time decreasing the energy consumption efficiency. The research presented in [13] proposed a recovery system based on the installation of pumps used as turbines (PATs). An annual estimation of the theoretical recoverable energy as well as the "ideal" pump for each point were proposed. The theoretical recovered energy value was $847,301 \mathrm{kWh} /$ year for a specific analyzed point. Besides, the characteristic curves of the PATs from a selected point were obtained, estimating an improvement in the sustainable indexes. From these calculations, a reduction in consumed energy of $1.50 \mathrm{kWh} / \mathrm{m}^{3}$ was estimated.

Paper [14] investigated capsule hydraulic transportation, a kind of low-carbon and environmentally friendly pipeline transportation technique. The flow velocity characteristics in the pipeline when the capsule was transported in a straight pipe section were simulated by adopting the renormalization group k- $\varepsilon$ turbulence model based on Fluent software, and experimentally verified. The results provided a theoretical basis for optimizing technical parameters of capsule hydraulic transportation.

The piped hydraulic transportation of tube-contained raw material represents a new long-distance transportation technology. This technology shows the advantages of high efficiency, energy savings, and environmental protection. According to the published literature, major limitations could be the speed, flow field, pressure field, and energy consumption of a single-pipe vehicle. Moreover, changes in vehicle spacing will affect the starting speed, flow field distribution, and pressure drop characteristics of the water flow within the pipeline. A numerical simulation was performed in [15] to study the hydraulic characteristics of stationary two-pipe vehicles under different spacings, comparing the results with physical experiments. The simulated results were in good agreement with the experiments. 
The aim of the study presented in [16] was to investigate the action mechanism of the rotor-stator interaction (RSI) on the transient flow field and hydrodynamic noise field inside the impeller of a jet centrifugal pumps (JCPs), and to optimize the effects of the guide vane on the hydraulic and hydroacoustic characteristics of the impeller. A computational fluid dynamics (CFD) method coupled with computational fluid acoustics (CFA) was applied to analyze the correlation between the guide vane and the flow/sound performances of the impeller. The orthogonal test method with the hydroacoustic performance of the impeller was used to optimize the structural parameters of the guide vane for the stability of the hydraulic performance of the JCP. The RSI led to a significant increase in the hydroacoustic level of the impeller, but was indispensable for improving the hydraulic performance of the pump.

The topic of paper [17] was the end clearance of the impeller of a high-speed electrical submersible pump (ESP). An ESP with a rotating speed of $6000 \mathrm{r} / \mathrm{min}$ was considered in this study. Numerical calculations were carried out for five different sizes of end clearance, ranging from $0.1 \mathrm{~mm}$ to $1.2 \mathrm{~mm}$, to obtain the performance and internal flow field under different situations. The simulated results were verified by the pump performance experiment. The increase of the end clearance led to a decrease in the head and efficiency of the ESP. The existence of the end clearance reduced the flow rate and caused free pre-whirl. Increasing the end clearance, the phenomenon of de-flow in the diffuser passage was aggravated. A reasonable recommended value of the end clearance was suggested.

Paper [18] studied the application of vortex pumps in sewer systems. Due to their outstanding anti-clogging ability, vortex pumps have gradually come into use in recent years. With sewage containing solid particles, vortex pumps encounter problems (e.g., partial clogging or overwork wear); therefore, it is particularly important to master the flow characteristics of solid particles in the vortex pump. In this paper, the discrete element model-computational fluid dynamics (DEM-CFD) coupled calculation method was introduced into the numerical simulation of vortex pumps, and fluid dynamic characteristics of particles with diameters of 1,2 , and $3 \mathrm{~mm}$ and concentrations of $1 \%$ and $5 \%$ were simulated.

In contrast to traditional installations based on water storage in artificial basins, free flow river turbines have a very low environmental impact due to their negligible effect on solid transport. Kinetic turbines with vertical axes are quite inexpensive, with almost zero impact on local fauna. In [19], the effect of an upstream deflector system mounted upstream of a twisted Savonius rotor inside a channel was investigated through numerical simulations and experimental tests. Numerical simulations were carried out using ANSYS FLUENT 17.0 software. This new design system contributes towards the more efficient use of flows in rivers and channels for electricity production in rural areas.

Crossflow turbines are a valuable choice for energy recovery in aqueducts due to their constructive simplicity and good efficiency under different head jump conditions. In [20], in reference to a specific case study, the authors firstly showed that the geometry of the most efficient impeller using blades with a traditional circular profile made with standard material would shortly lead to their mechanical failure. A methodology for fully coupled fluid dynamic and mechanical optimization of the blade cross-section was then proposed. A linear variation of the curvature of the external blade surface was assumed, along with an iterative use of 2D computational fluid dynamic (CFD) and 3D structural finite element method (FEM) simulations. The proposed methodology was applied to the design of a power recovery system (PRS) turbine operating in a water transport network.

Funding: This research received no external funding.

Acknowledgments: The author would like to acknowledge all of the contributions to the Special Issue, the time invested by each author, and the anonymous reviewers and the editorial managers who contributed to the development of the articles in this Special Issue. The author is very happy with the review process and management.

Conflicts of Interest: The author declares no conflict of interest. 


\section{References}

1. Rong, Y.; Zhang, T.; Peng, L.; Feng, P. Three-Dimensional Numerical Simulation of Dam Discharge and Flood Routing in Wudu Reservoir. Water 2019, 11, 2157. [CrossRef]

2. Hien, L.T.T.; Van Chien, N. Investigate Impact Force of Dam-Break Flow against Structures by Both 2D and 3D Numerical Simulations. Water 2021, 13, 344. [CrossRef]

3. Cunha, A.H.F.; Fragoso, C.R.; Tavares, M.H.; Cavalcanti, J.R.; Bonnet, M.-P.; Motta-Marques, D. Combined Use of High-Resolution Numerical Schemes to Reduce Numerical Diffusion in Coupled Nonhydrostatic Hydrodynamic and Solute Transport Model. Water 2019, 11, 2288. [CrossRef]

4. Cunha, A.H.F.; Fragoso, C.R., Jr.; Chalegre, C.L.B.; Motta-Marques, D. Improvement of Non-Hydrostatic Hydrodynamic Solution Using a Novel Free-Surface Boundary Condition. Water 2020, 12, 1271. [CrossRef]

5. Zhou, H.; Wang, G.; Jia, C.; Li, C. A Novel, Coupled CFD-DEM Model for the Flow Characteristics of Particles Inside a Pipe. Water 2019, 11, 2381. [CrossRef]

6. Lee, W.-D.; Mizutani, N.; Hur, D.-S. 2-D Characteristics of Wave Deformation Due to Wave-Current Interactions with Density Currents in an Estuary. Water 2020, 12, 183. [CrossRef]

7. Li, H.; Ren, H.; Qiu, S.; Wang, C. Physics-Based Simulation of Ocean Scenes in Marine Simulator Visual System. Water 2020, 12, 215. [CrossRef]

8. Liu, S.; Ma, F.; Zhao, H.; Guo, J.; Duan, X.; Sun, Q. Numerical Investigation of a Hydrosplitting Fracture and Weak Plane Interaction Using Discrete Element Modeling. Water 2020, 12, 535. [CrossRef]

9. Huang, X.; Wang, L.-1.; Xu, J. Numerical Study on Dynamical Structures and the Destratification of Vertical Turbulent Jets in Stratified Environment. Water 2020, 12, 2085. [CrossRef]

10. Ghaderi, A.; Dasineh, M.; Aristodemo, F.; Aricò, C. Numerical Simulations of the Flow Field of a Submerged Hydraulic Jump over Triangular Macroroughnesses. Water 2021, 13, 674. [CrossRef]

11. Li, Y.; Gao, Y.; Jia, X.; Sun, X.; Zhang, X. Numerical Simulations of Hydraulic Characteristics of A Flow Discharge Measurement Process with A Plate Flowmeter in A U-Channel. Water 2019, 11, 2382. [CrossRef]

12. Pérez-Sánchez, M.; Sánchez-Romero, F.J.; Ramos, H.M.; López-Jiménez, P.A. Improved Planning of Energy Recovery in Water Systems Using a New Analytic Approach to PAT Performance Curves. Water 2020, 12, 468. [CrossRef]

13. Camilo Rosado, L.E.; López-Jiménez, P.A.; Sánchez-Romero, F.-J.; Conejos Fuertes, P.; Pérez-Sánchez, M. Applied Strategy to Characterize the Energy Improvement Using PATs in a Water Supply System. Water 2020, 12, 1818. [CrossRef]

14. Li, F.; Li, Y.; Sun, X.; Yang, X. Numerical Simulation of Flow Velocity Characteristics during Capsule Hydraulic Transportation in a Horizontal Pipe. Water 2020, 12, 1015. [CrossRef]

15. Jia, X.; Sun, X.; Li, Y. Numerical Simulation of the Flow Field Characteristics of Stationary Two-Pipe Vehicles under Different Spacings. Water 2020, 12, 2158. [CrossRef]

16. Guo, R.; Li, R.; Zhang, R.; Han, W. The Action Mechanism of Rotor-Stator Interaction on Hydraulic and Hydroacoustic Characteristics of a Jet Centrifugal Pump Impeller and Performance Improvement. Water 2020, 12, 465. [CrossRef]

17. Zhou, L.; Wang, W.; Hang, J.; Shi, W.; Yan, H.; Zhu, Y. Numerical Investigation of a High-Speed Electrical Submersible Pump with Different End Clearances. Water 2020, 12, 1116. [CrossRef]

18. Gao, X.; Shi, W.; Shi, Y.; Chang, H.; Zhao, T. DEM-CFD Simulation and Experiments on the Flow Characteristics of Particles in Vortex Pumps. Water 2020, 12, 2444. [CrossRef]

19. Mosbahi, M.; Lajnef, M.; Derbel, M.; Mosbahi, B.; Aricò, C.; Sinagra, M.; Driss, Z. Performance Improvement of a Drag Hydrokinetic Turbine. Water 2021, 13, 273. [CrossRef]

20. Sinagra, M.; Picone, C.; Aricò, C.; Pantano, A.; Tucciarelli, T.; Hannachi, M.; Driss, Z. Impeller Optimization in Crossflow Hydraulic Turbines. Water 2021, 13, 313. [CrossRef] 